\title{
Épigenèse et littérature pour enfants : à propos des contes des frères Grimm
}

Vanessa Joosen, Vincent Neyt et Dirk Van Hulle

\section{(2) OpenEdition}

1 Journals

Édition électronique

URL : http://journals.openedition.org/genesis/3922

DOI : 10.4000/genesis.3922

ISSN : 2268-1590

Éditeur :

Presses universitaires de Paris Sorbonne (PUPS), Société internationale de génétique artistique littéraire et scientifique (SIGALES)

Édition imprimée

Date de publication : 1 juin 2019

Pagination : 21-36

ISSN : 1167-5101

Référence électronique

Vanessa Joosen, Vincent Neyt et Dirk Van Hulle, «Épigenèse et littérature pour enfants : à propos des contes des frères Grimm », Genesis [En ligne], 48 | 2019, mis en ligne le 01 juin 2020, consulté le 24 janvier 2021. URL : http://journals.openedition.org/genesis/3922 ; DOI : https://doi.org/10.4000/ genesis.3922 


\title{
Épigenèse et littérature pour enfants À propos des contes des frères Grimm
}

\author{
Vanessa Joosen, Vincent Neyt, Dirk Van Hulle
}

$\mathrm{S}$ ouvent la genèse d'une œuvre littéraire continue après sa publication. Ce type de genèse ne fait pas partie de ce que Raymonde Debray Genette a appelé «endogenèse »; elle n'appartient plus à l'intérieur (endo-) de la genèse, mais se situe «après». C'est pourquoi Rudolf Mahrer et les contributeurs d' «Après le texte» (Genesis, $\left.{ }^{\circ} 44\right)$ l'appellent «genèse post-éditoriale ${ }^{1}$ ». Comme le note Mahrer : «La polarité entre texte et avant-texte se fait moins claire lorsqu'on prend en considération des documents qui ne sont génétiques qu'a posteriori ${ }^{2}$.»

Nous sommes parfaitement d'accord sur le fait que la critique génétique a eu tendance à privilégier ce qui précède le moment du «bon à tirer». Parce que les transformations du texte au-delà de ce point prennent place dans un environnement public, leur logique ne correspond plus à celle des procès d'écriture de l'avant-texte, selon Pierre-Marc de Biasi ${ }^{3}$. Longtemps, cette autre logique semble avoir conduit à considérer que la continuation de la genèse après le moment du «bon à tirer» n'appartient pas au domaine de la critique génétique, mais plutôt à celui de la critique textuelle et à la philologie. Mais si les écrivains traduisent leurs propres écrits (comme Samuel Beckett), ou si la réception de leur œuvre les conduit à de constantes révisions (comme dans le cas de Charles Darwin), l'on pourrait avancer que la genèse continue bel et bien après «le bon à tirer». Par suite, depuis 2007, en sus des termes d'exogenèse et d'endogenèse proposés par Raymonde Debray Genette, les généticiens du Centre pour la génétique des manuscrits de l'Université d'Anvers ont utilisé celui d'épigenèse pour se référer à la continuation de la genèse après publication 4 .

Si nous avons choisi de travailler avec ce terme, y compris dans cette contribution, c'est qu'il fait fond de l'apparente polarité entre texte et avant-texte, mais aussi d'autres dynamiques subtiles entre l'exo-, l'endo- et l'épigenèse, suggérant un modèle triangulaire ${ }^{5}$. Celui-ci fonctionne comme le triangle des couleurs de James Clerk Maxwell, fondé sur la combinaison des trois couleurs de base à chacun de ses sommets. Le modèle triangulaire représente la genèse non seulement comme un développement linéaire (avant et après la publication), mais aussi comme une gamme d'actions génétiques, une structure interconnectée où, en n'importe

1. Rudolf Mahrer, «La plume après le plomb : Poétique de la réécriture des œuvres déjà publiées », Genesis, ${ }^{\circ} 44$, 2017, p. $17-38$, p. 22.

2. Mahrer, «La plume après le plomb», art. cit., p. 18.

3. Pierre-Marc de Biasi, «Qu'est-ce qu' un brouillon? Le cas Flaubert : essai de typologie fonctionnelle des documents de genèse», dans M. Contat et D. Ferrer (dir.), Pourquoi la critique génétique? Méthodes, théories, Paris, CNRS éditions, 1998, p. 31-60, p. 43.

4. Voir Dirk Van Hulle, De kladbewaarders, Nijmegen, Vantilt, 2007 - une introduction à la critique génétique en trois parties : «exogenèse», «endogenèse » et «épigenèse».

5. Pour une discussion plus détaillée, voir Dirk Van Hulle, «Modern Manuscripts» dans The Oxford Encyclopedia of Literary Theory (à venir). 
quel point, il se peut que du matériel exogénétique colore l'endogenèse ou l'épigenèse. Dans le contexte du présent article sur la comparaison de versions, le modèle triangulaire invite à considérer les variantes non comme des différences textuelles entre éditions, qui s'écartent du texte ne varietur ou «définitif » (un terme qui n'est plus d'usage en philologie), mais comme des éléments d'un processus génétique long et complexe, reliant l'épigenèse à l'exogenèse.

(1) La relation complexe entre l'exogenèse et l'endogenèse avait déjà été relevée par Debray Genette en 1977 lorsqu'elle inventa ces termes dans son essai «Genèse et poétique : esquisse de méthode» :

Il y a une grande distance apparente, dans les procès évolutifs, entre ce que l'on pourrait appeler les effets d'exogenèse et ceux d'endogenèse. Le terme d'exogenèse pourrait paraître dangereux aux puristes de la textualité, s'il signifiait qu'on sortît des documents écrits. [...] Il est vrai qu'il n'est pas facile d'éviter le «ceci cause cela», le balzacien « voici pourquoi», la présentation de l'œuvre comme un reflet de la vie [...] Par conséquent, nous ne pouvons nous placer au départ que du point de vue du «donné» écrit, dans la mesure où il a été conservé; nous ne pouvons qu'essayer d'analyser, de structurer, en évitant le plus possible l'herméneutique, l'ensemble des signifiants conservés6.

Depuis le début, Debray Genette reconnaît que «les phénomènes d'endogenèse sont parfois difficiles à distinguer de ceux d'exogenèse ${ }^{7}$ », puisque dès qu'un auteur prend une note en lisant un livre, celle-ci pourrait déjà appartenir au procès endogénétique. La relation entre exo- et endogenèse est dépeinte par Nicolas Cavaillès à travers une métaphore liquide :

La rivière de l'exogenèse se jette dans le fleuve de l'endogenèse, tout élément exogénétique étant progressivement incorporé au texte en cours jusqu'à s'y fondre et y suivre un destin commun avec les éléments endogénétiques qui l'environnent ${ }^{8}$.

(2) La relation entre l'endogenèse et l'épigenèse a été décrite comme un passage du texte d'un état fluide à un état figé, dont le «bon à tirer» constitue, selon la traduction anglaise de l'article de Pierre-Marc de Biasi, «the decisive moment when what had been in a pliable and mobile manuscript state up to that point becomes fixed in the frozen shape of a published text ${ }^{9}$ » - une métaphore qui a été mise en question et nuancée 10 parce que, au moins du point de vue de son auteur, le texte reste souvent dans un état liquide et modifiable ${ }^{11}$.

(3) Le troisième lien, entre exogenèse et épigenèse, est un élément crucial dans la « genèse post-éditoriale ». Quand, par exemple, un auteur dramatique a l'occasion de diriger sa propre

6. Raymond Debray Genette, «Génétique et poétique : esquisse de méthode», Littérature, n² 28, 1977, p. 19-39; p. 24. En ligne : https://www.persee.fr/doc/litt_0047-4800_1977_num_28_4_2073. Voir aussi Pierre-Marc de Biasi, «Qu'est-ce qu'un brouillon?», op. cit., p. 45-46.

7. Ibid., p. 27.

8. Nicolas Cavaillès, «L’Exogenèse : Le cas du Précis de décomposition de Cioran », Recto/Verso, n ${ }^{\circ}$ 1, 2007. En ligne : <http://www.revuerectoverso.com/spip.php?article48\#nb2>.

9. Pierre-Marc de Biasi, «What is a Literary Draft. Toward a Functional Typology of Genetic Documentation», I. Wassenaar (trad.), Yale French Studies, nº 89 (1996), p. 37. La métaphore est moins saisissante dans la version française, mais l'idée de fixation est la même : «Ce qui n'était jusqu'alors qu'un état toujours mobile et changeant du manuscrit va se fixer sous la forme arrêtée d'un texte publié.»

10. Dirk Van Hulle, «Essai de critique épigénétique», dans P.-M. de Biasi et al. (dir.), L'Euvre comme processus, Paris, CNRS Éditions, 2017, p. 415-430.

11. Voir aussi John Bryant, The Fluid Text: A Theory of Revision and Editing for Book and Screen, Ann Arbor, University of Michigan Press, 2002. 
pièce et décide d'opérer des changements, certaines de ses modifications «post-éditoriales » peuvent avoir été inspirées par quelque chose qu'il a lu12. La métaphore de la rivière, proposée par Cavaillès, s'applique aussi à ce phénomène, qui n'est évidemment pas limité à la littérature, comme l'attestent par exemple les nombreuses révisions apportées par Darwin à chacune des six éditions de The Origin of Species parues de son vivant. Par ces révisions, le scientifique a souvent réagi à des relecteurs critiques, ce qui constitue une forme d'exogenèse épigénétique ou d'épigenèse exogénétique.

Ce phénomène est particulièrement pertinent dans la littérature pour enfants, comme nous aimerions le montrer dans cet article, à partir du cas des contes des Grimm. Comme le note Christa Kamenetsky, il échappa à la première réception des recueils de contes qu'il n'existait pas de Urmärchen ( «contes originaires») et que les Grimm étaient constamment aux prises avec des versions et des variantes ${ }^{13}$. La seconde édition des Kinder-und Hausmärchen contenait non seulement de nouveaux contes mais intégrait également de nouvelles variantes ${ }^{14}$. C'est Wilhelm qui, à partir de 1819, assuma principalement la responsabilité des révisions et de la combinaison des fragments de conte : «Il n'insérait des fragments de conte dans un autre conte que si le conte populaire principal le convainquait essentiellement de sa substance», note Kamenetsky ${ }^{15}$. Wilhelm Grimm s'expliqua sur cette procédure dans la préface de la seconde édition, recourant à une métaphore corporelle pour décrire l'acte d' «incorporation » du nouveau matériel exogénétique : «Ce que nous avons gagné jusqu'à maintenant pour notre collection, nous voulions l'incorporer dans cette seconde édition 16 . $\gg$ Cette pratique montre à quel point le matériel exogénétique rencontré par les frères Grimm après la première édition des Kinder- und Hausmärchen continua à jouer un rôle majeur dans l'épigenèse de leur recueil.

En tant que discours contribuant à forger la vision du monde des générations futures, les implications idéologiques des livres pour enfants ont été particulièrement scrutées. En conséquence, les passages problématiques des classiques de la littérature enfantine ont été révisés. Par exemple, après qu'Eleanor Cameron a vertement critiqué Charlie and the Chocolate Factory de Roald Dahl pour son racisme, les illustrations des Oompa-Loompas en Pygmées noirs ont été remplacées dans l'édition suivante ${ }^{17}$. Par ailleurs, les contenus et les formes de la littérature pour enfants sont conditionnés par les représentations des enfants-lecteurs que se font les adultes, ceux-ci essayant d'évaluer ce qui intéresse ceux-là, et ce à quoi ils peuvent faire face sur le plan cognitif et psychologique. La vision qu'ont les adultes de ce que peut

12. Voir notamment l'exemple de la lecture de Samuel Beckett par Matthias Claudius, dans Dirk Van Hulle, «Essai de critique épigénétique», art. cit., p. 426-427.

13. Christa Kamenetsky, The Brothers Grimm and Their Critics : Folktales and the Quest for Meaning, Athens, OH : Ohio University Press, 1992, p. 163.

14. Ibid., p. 163.

15. Ibid., p. 166.

16. Wilhelm Grimm, «Vorrede», dans Kinder- und Hausmärchen. Zweite vermehrte und verbesserte Auflage, Berlin, Reimer, 1819, p. xiv; nous soulignons.

17. Lucy Mangan, «Charlie and the Chocolate Factory at $50 »$, The Guardian, 30 août 2014. <https://www.theguardian. com/books/2014/aug/30/charlie-and-the-chocolate-factory-50-years-roald-dahl-quentin-blake $>$. Consulté le 1 er septembre 2017.

\section{Épigenèse et littérature pour enfants}


être une lecture parfaite pour les enfants diverge et évolue : les différences culturelles et les changements historiques dans la représentation de l'enfance sont évidents et, même au sein d'une même culture, les adultes ne s'accordent pas forcément sur ce qui constitue une lecture appropriée aux jeunes ${ }^{18}$. En outre, la poétique d'un auteur de littérature pour enfants évolue au cours de sa carrière, sous l'influence de facteurs aussi variés que des expériences nouvelles avec des enfants (par exemple, le fait d'en avoir lui-même), les réactions de lecteurs jeunes ou adultes, les rencontres avec d'autres styles littéraires pour enfants, l'acquisition de nouvelles compétences littéraires, un changement d'éditeur et ainsi de suite ${ }^{19}$. Le changement de poétique et la modification des représentations de l'enfance sont parfois si forts qu'ils motivent les auteurs à réviser des œuvres déjà publiées, parfois même plusieurs fois.

Dans cet article, nous nous proposons de discuter quelques révisions épigénétiques des frères Grimm à la lumière de la conception de l'enfance du début du XIXe siècle. Notre objectif est triple. D'abord, nous envisagerons les recueils des Grimm comme un cas particulièrement pertinent pour illustrer l'effet de conceptions opposées de l'enfance sur des révisions épigénétiques, ainsi que la complexité des négociations au cours de ce processus ${ }^{20}$. Deuxièmement, nous aimerions montrer que des outils numériques peuvent aider les chercheurs en génétique à développer leurs observations concernant les étapes de l'épigenèse des contes de Grimm, qui ne comprend pas moins de sept (grandes) éditions. Troisièmement, partant de l'observation que les conceptions de l'enfance qui influencent les révisions ont été édictées par des adultes, nous montrerons que des outils numériques peuvent aider à introduire la genèse de Die Kinder- und Hausmärchen auprès de ceux-là mêmes qui sont les sujets de ce discours : les jeunes lecteurs. Ce faisant, nous renouons avec de récents travaux dans le champ de la littérature jeunesse, qui insistent sur l'action potentielle des enfants dans la production ${ }^{21}$ et la compréhension d'artefacts culturels qui leur sont destinés22.

Plusieurs mythes romantiques ont été associés aux Kinder- und Hausmärchen de Jacob et Wilhelm Grimm depuis la première publication du recueil en 181223. L'imagination populaire se représente les frères Grimm en scribes fidèles parcourant la campagne allemande et invitant les paysans illettrés à partager leurs histoires. Par suite, on a tenu les contes de Grimm pour d'authentiques contes populaires allemands qui - en raison de leur longue tradition orale capturent les vérités humaines qui transcendent le temps et l'espace.

Ces mythes se sont montrés plutôt persistants, en dépit d'un riche corpus de recherches qui jette une lumière différente sur la méthode des Grimm. Les références données en notes par

18. Zohar Shavit, Poetics of Children's Literature, Athens, University of Georgia Press, 1986.

19. Pour une autre étude de cas, celle d'un auteur dont la conception de l'enfance est plus contemporaine que celle des frères Grimm, voir Vanessa Joosen, «Allez Changez ! / En nog een keer! : De genese van Suzanne Dantine en Wespennest », Literatuur zonder leeftijd, n 95, 2014, p. 107-134.

20. À cet égard, comme le note Cyrille François, les Grimm s'écartent, par exemple, d'Andersen, puisqu'ils ont continué à réviser leurs éditions «en les modifiant constamment afin d'approcher, par une élaboration littéraire, la simplicité et la pureté qu'ils considèrent propres aux contes populaires». Cyrille François, «Andersen trouve-t-il son conte?», Genesis, n 44, 2017, p. 65.

21. Marah Gubar, Artful Dodgers: Reconceiving the Golden Age of Children's Literature, Oxford, Oxford University Press, 2009.

22. Justyna Deszcz-Tryhubczak, «Using Literary Criticism for Children’s Rights: Toward a Participatory Research Model of Children's Literature Studies », The Lion and the Unicorn, n 2, 2016, p. 215-231.

23. Jacob et Wilhelm Grimm, Die Kinder- und Hausmärchen, H. Rölleke (dir.), 3 vol., Stuttgart, Reclam, 2001. 
les frères Grimm montrent qu'ils se sont fondés en partie sur des sources écrites. Des travaux sur les marginalia de leurs exemplaires personnels ont révélé que plusieurs de leurs contes les plus célèbres, tels que «Blanche Neige» et «Le Petit Chaperon rouge», proviennent de jeunes femmes instruites par leur entourage bourgeois ${ }^{24}$. D' autres recherches philologiques sur les manuscrits et les éditions de Die Kinder- und Hausmärchen (1812-1857) ont exposé les multiples manières par lesquelles les frères Grimm ont adapté les contes sur le plan du contenu et du style, et comment Wilhelm Grimm en particulier révisait les contes à chaque édition. La période d'activité éditoriale la plus intense peut être située dans les années qui précédèrent et suivirent la publication du premier volume, en 1812.

Dans une première étape, débutant en 1807, les Grimm réunirent des contes et combinèrent les variantes de chaque histoire pour construire ce qu'ils considéraient comme leur forme idéale, selon un processus appelé quelque peu paradoxalement «contamination». Siegfried Neumann le décrit comme suit : «Lorsqu'ils étaient confrontés à plusieurs versions du même conte, Wilhelm s'efforçait dans chaque cas de choisir le meilleur en termes de contenu et de récit. Et lorsqu'il pensait possible d'étendre ou d' "améliorer" ladite version à l'aide d'une autre version, il le faisait ${ }^{25}$.» Comme nous l'avons brièvement expliqué plus haut, Wilhelm Grimm pratiquait la contamination tout au long du processus génétique de Die Kinder- und Hausmärchen, y compris au cours des révisions épigénétiques. Par ailleurs, quand les Grimm se lancèrent dans leur projet de contes, les enfants n'étaient pas leur première cible; après la publication du premier volume pourtant, ils reçurent des critiques de la part d'adultes qui, ayant lu les contes à des enfants, les avaient trouvés inappropriés. Achim von Arnim, celui qui a donné envie aux Grimm de commencer leur œuvre, leur suggéra d'adapter les contes «au goût et à l'âge» des enfants26. Que penser, par exemple, de l'histoire dans laquelle des enfants jouent au boucher, et où l'un tranche la gorge de l'autre? Tout aussi controversée fut l'histoire de la renarde qui se languit désespérément de son compagnon, un renard à neuf queues qu'elle croit mort. Aucun renard s'offrant en remplacement ne satisfait ses besoins. De si cruelles histoires, ou si sexuellement suggestives, ont été jugées inadaptées pour les jeunes. En plus de ces voix critiques, les Grimm devaient faire face aux maigres ventes de leur première édition, tandis que le recueil Histoires populaires allemandes (1823) d'Edgar Taylor et David Jardine, constitué d'une petite sélection illustrée de contes de Grimm adaptés et sélectionnés pour les enfants, connut un succès immédiat à l'étranger 27.

Tandis que, d'abord, les Grimm «ont insisté sur le fait que les enfants devaient lire les contes populaires comme ils lisent la Bible, sans embellissement, sans abrégement, ou altérations concernant leur substance épique 28 », l'évolution épigénétique de leur recueil suggère que les pratiques éditoriales de Wilhelm répondaient à l'idée que les contes devaient s'adapter davantage aux enfants - ou du moins correspondre à ce que les parents et les éducateurs

24. Heinz Rölleke, Die älteste Märchensammlung der Brüder Grimm, Cologne/Genève, Fondation Martin Bodmer, 1975 .

25. Siegfried Neumann, «The Brothers Grimm as Collectors and Editors of German Folktales», dans D. Haase (dir.), The Reception of Grimm's Fairy Tales: Responses, Reactions, Revisions, Detroit, Wayne State UP, 1993, p. 32.

26. Kamenetsky, op. cit., p. 193.

27. Martin Sutton, The Sin-Complex: A Critical Study of English Versions of the Grimms'Kinder- und Hausmärchen in the Nineteenth Century, Kassel, Brüder Grimm Gesellschaft, 1996.

28. Kamenetsky, op. cit., p. 193. 


\section{Recherche épigénétique assistée par ordinateur}

considéraient comme le plus approprié aux enfants. Alors que les Grimm restèrent fidèles à leur conviction que ces derniers ne devaient pas être protégés du mal, ils supprimèrent les contes particulièrement cruels et suggestifs, comme les deux mentionnés plus haut, et en adaptèrent plusieurs autres afin de les rendre plus acceptables en tant que littérature pour enfants 29 . À partir de 1825, ils commencèrent également à publier de «petites éditions » sélectives, spécifiquement adressées au jeune public. À chaque nouvelle édition, Wilhelm peaufinait les contes par de petits changements, renforçant notamment leurs propriétés génériques, effaçant des incohérences, explicitant des références aux valeurs chrétiennes et des germanismes. Des critiques tels que Rölleke ${ }^{30}$, Zipes ${ }^{31}$, Bottigheimer ${ }^{32}$ et Tatar ${ }^{33}$ ont mis en évidence le préjugé idéologique de ces éditions, en même temps qu'ils reconnaissaient à Wilhelm le mérite d'une réécriture des contes populaires qui rendait ceux-ci non seulement recevables pour les parents allemands du XIXe siècle, mais aussi à même de toucher des lecteurs bien au-delà de leur contexte original de publication.

Un riche ensemble de travaux sur la pratique rédactionnelle des Grimm est déjà disponible. Pourtant, les mythes entourant leur recueil sont si tenaces que le besoin de communiquer les faits et les observations persiste lui aussi, et manifestement cet effort doit être poursuivi pour chaque nouvelle génération de lecteurs et d'enseignants. Les outils numériques peuvent y contribuer, comme nous allons le montrer plus loin. De plus, l'étendue de l'activité éditoriale des Grimm est si vaste que l'analyse numérique peut également contribuer à produire de nouvelles observations. Plusieurs outils des Humanités numériques, de différents niveaux de complexité, peuvent être employés pour ajouter ce que Matthew Jockers appelle une perspective «macro-analytique» à la lecture microtextuelle des éditions des Grimm ${ }^{34}$. Des programmes de modélisation des thèmes comme LDA et des outils de concordance comme Antconc peuvent aider les chercheurs à cartographier les thématiques et les changements idéologiques dans Die Kinder- und Hausmärchen en mettant en évidence les mots typiquement associés dans les différentes éditions. En étiquetant les parties du discours et le genre des locuteurs, et en appliquant ensuite un simple comptage de mots, la progressive réduction au silence des personnages (féminins) ${ }^{35}$ peut être examinée à une large échelle. Des programmes de stylométrie comme $\mathrm{R}^{36}$ peuvent regrouper les contes sur la base de leur similarité stylistique, permettre de visualiser la mise en place de ce qu' on appelle la «Gattung Grimm» ou le style des Grimm, et d'orienter les chercheurs vers les contes qui ont échappé à l'homogénéisation stylistique.

\footnotetext{
29. Maria Tatar, The Hard Facts of the Grimms'Fairy Tales, 2e éd., Princeton, Princeton UP, 2003.

30. Voir plus haut, note 24 .

31. Jack Zipes, The Brothers Grimm, 2e éd., New York, Palgrave Macmillan, 2002.

32. Ruth B. Bottigheimer, Grimms'Bad Girls \& Bold Boys: The Moral \& Social Vision of the Tales, New Haven, Yale University Press, 1987.

33. Voir plus haut, note 29.

34. Matthew L. Jockers, Macroanalysis: Digital Methods and Literary History, University of Illinois Press, 2013.

35. Ruth B. Bottigheimer, op. cit.

36. Maciej Eder, Jan Rybicki et Mike Kestemont, «Stylometry with R: A Package for Computational Text Analysis », The R Journal, 2016. En ligne : < https://journal.r-project.org/archive/2016-1/eder-rybicki-kestemont.pdf >.
} 
Pour faciliter nos recherches épigénétiques, nous recourons à des développements récents dans le champ de la collation automatique. Avec CollateX ${ }^{37}$, nous avons comparé quatre contes des frères Grimm : «Rose Bruyère», «Blanche Neige», «Hansel et Gretel» et «Le Petit Chaperon rouge ». CollateX permet la comparaison d'un nombre illimité de versions d'un texte sans que l'une d'entre elles serve de base. Le programme peut être intégré à une application web via son service REST, et fournit cinq formats de sortie (segmentation parallèle en TEI P5, graphe de variants, table d'alignement [JSON], GraphML et GraphViz). Contrairement à MEDITE et à Juxta, CollateX ne vise que des utilisateurs ayant une certaine compétence technique, puisqu'il ne comporte pas d'interface graphique d'utilisation. Les collations, comportant jusqu'à dix versions de chaque conte dans notre projet pilote, fonctionnent actuellement comme un outil de recherche interne 38 .

Les plus anciennes versions de «Rose Bruyère », «Hansel et Gretel » et «Blanche Neige » sont déjà présentes dans le manuscrit d'Ölenberg (1810). Le «Petit Chaperon rouge» apparaît initialement dans la première édition des Kinder- und Hausmärchen en 1812. Des versions de chacun de ces quatre contes ont été publiées dans toutes les éditions de Die Kinder- und Hausmärchen de 1812 à 1857, c'est-à-dire sept grandes éditions (1812/5, 1819, 1837, 1840, $1843,1850,1857)$ et dix petites $(1825,1833,1836,1829,1841,1844,1847,1850,1853$, 1858), soit un total de 17 éditions parues de leur vivant. Dans le projet actuel, nous avons inclus les versions du manuscrit de 1810 lorsqu'elles étaient disponibles, ainsi que les sept versions publiées dans les grandes éditions, en écartant pour l'instant les petites.

Notre projet pilote est un outil de collation assistée par ordinateur. Sur la base de la transcription en texte brut du manuscrit, des grandes éditions et des exemplaires personnels annotés par les Grimm, une comparaison numérique a été générée avec CollateX. Cette comparaison a servi de point de départ pour l'élaboration manuelle d'un appareil critique électronique, où les mots correspondant sont mis en relation et les différences soulignées. À partir de ce fichier maître ont été générées des comparaisons de toutes les combinaisons possibles comprenant jusqu'à dix versions : elles forment la base de notre site internet pilote.

Les utilisateurs peuvent lire n'importe quelle version de chacun des contes, aux côtés de reproductions de leur édition d'origine, ou choisir n'importe quel nombre de versions pour générer un appareil de comparaisons. Au sommet de la table, en cliquant sur «In numbers » on fait apparaître des statistiques de cette comparaison. À quel point ces textes sont-ils similaires ? Combien de mots ont-ils été ajoutés ou changés? Toutes les versions sont alignées dans une table verticale. Le fond rose et les caractères rouge violet dans certaines cellules signifient que les mots de cette cellule diffèrent des mots de la cellule correspondante dans la version précédente (fig. 1).

37. <https://collatex.net >.

38. Un site public est en développement. Pour l'intégration de CollateX dans une édition numérique, voir par exemple le «Beckett Digital Manuscript Project». Cf. <www.beckettarchive.org >. 


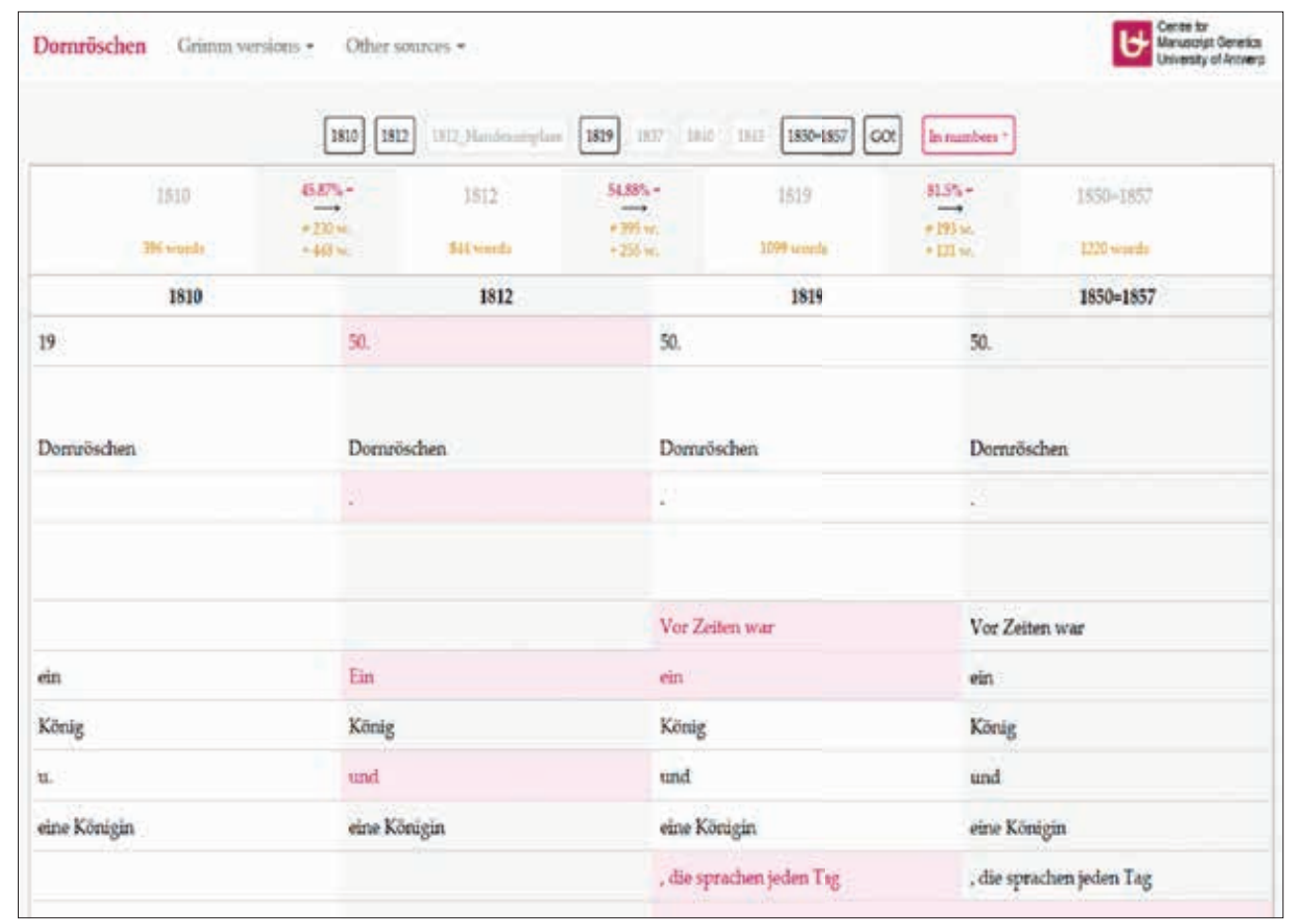

Fig. 1 : Copie d'écran d'une comparaison de quatre versions de « Rose Bruyère».

Comparées à ce qu'on appelle les «appareils de variantes» ou les appareils critiques des livres, dans lesquels chaque variante d'un certain texte est présentée séparément pour faciliter la recherche philologique, les collations numériques présentent des avantages variés, qui vont bien au-delà d'une précieuse économie de temps. Tout d'abord, elles permettent d'estimer en un coup d'œil l'intensité du processus éditorial (nombre et ampleur des changements). Ainsi, la comparaison ci-dessus montre clairement que «Rose Bruyère» a subi plus de changements entre 1810 et 1812 et entre 1812 et 1819 qu'entre 1819 et la version finale de 1857 . Dans les premières étapes, seule la moitié du conte est restée stable, alors que dans les phases ultérieures, le nombre de changements est considérablement plus bas.

La vue d'ensemble montre également comment le conte s'est progressivement allongé. Les comparaisons pourraient servir de point de départ pour des dénombrements de mots d'une plus fine granularité, introduisant une distinction, par exemple, entre des changements purement orthographiques et de réels remplacements de mots.

\section{Mise en œuvre des collations numériques}

Les collations numériques peuvent en outre aider à affiner les conclusions des éditions établies avec des moyens philologiques plus traditionnels. Les chercheurs peuvent ainsi vérifier si leurs résultats sont valables à une large échelle ou seulement pour le cas considéré. Par exemple, Ruth Bottigheimer explique comment les choix de Wilhelm ont changé la position idéologique de certains contes, concernant, notamment, la religion. 
En littérature populaire, écrit-elle, avant les Grimm [...] le christianisme et les éléments chrétiens sont remarquables par leur absence. [Une] analyse de l'histoire éditoriale des Contes de Grimm montre clairement que c'était Wilhelm Grimm lui-même qui glaçait le gâteau, pour ainsi dire, avec des exclamations chrétiennes, dont très peu existaient dans les versions de 1812 ou 181939 .

De cette observation et du chapitre qui suit nous dérivons un nombre de mots-clés associés, que nous avons ensuite utilisés pour interroger la comparaison de toutes les versions. Dans ce cas, nous avons mené l'enquête sur le manuscrit d'Ölenberg, les sept versions des grandes éditions et les deux copies personnelles des auteurs de «Blanche Neige» avec les mots-clés : «Gott», «göttlich», «gottlos», «Teufel», «teuflisch», «Gebet», «beten», « sich befehlen», «Himmel», «Hölle», «heilig», «fromm», «Sünde» et «sündig», signifiant respectivement Dieu, divin, impie, diable, diabolique, prière, prier (deux fois), ciel, enfer, sacré, pieux, péché et pécheur. Les copies d'écran en page 30 montrent au premier coup d'œil que la collation corrobore mais aussi nuance les découvertes de Bottigheimer, du moins en ce qui concerne «Blanche Neige». Le manuscrit ne contient aucune référence religieuse, de sorte que l'affirmation de Bottigheimer selon laquelle l'aspect religieux des contes publiés reflète l'intervention idéologique de Wilhelm Grimm résiste - du moins si l'on suppose que le manuscrit reflète la version de «Blanche Neige» où les Grimm sont le moins intervenus 40 . Le renforcement graduel mais régulier des contenus chrétiens dans les contes est aussi apparent, mais pour «Blanche Neige», il commence plus tôt que ne le suggère Bottigheimer. L'exclamation «ei du mein Gott» [oh mon Dieu] (fig. 2), que les nains prononcent deux fois lorsqu'ils trouvent Blanche Neige, était déjà présente dans la première édition. La vilaine reine est déjà traitée d' «impie» en 1812, avec une nouvelle occurrence ajoutée en 1840 (fig. 3). La phrase «befahl sich Gott» [pria Dieu] dans la scène où Blanche Neige va au lit dans la maison des sept nains a été ajoutée en 1819, et resta dans toutes les éditions suivantes (fig. 4). De manière similaire, Blanche Neige mentionne Dieu quand elle se réveille dans son cercueil de verre pour la première fois en 1819 .

Toutes les références religieuses ajoutées à «Blanche Neige» ont été retenues jusqu'au bout. Ces changements attestent non seulement des croyances personnelles de Wilhelm, mais peuvent aussi être interprétés comme le reflet des représentations de l'enfant au début du XIX ${ }^{\mathrm{e}}$ siècle : des pédagogues éclairés considèrent que le merveilleux et les contes de fées ne conviennent pas aux jeunes, car ils pourraient les distraire de récits plus utiles - didactiques et religieux. La dimension chrétienne viendrait en fait contribuer à élargir l'audience des contes des Grimm : la dernière édition de «Blanche Neige», par exemple, peut être lue comme la lutte entre les pieux et les impies, célébrant la victoire des premiers et la cruelle défaite des seconds.

La combinaison de la collation et de l'outil de recherche permet d'apporter des raffinements et des nuances à notre connaissance des sources, mais ajoute-t-elle de nouvelles observations ? On trouvera une réponse à cette question du côté de l'absence de certains résultats qui peut faire surgir de nouvelles questions de recherche. Par exemple, une même recherche des termes religieux dans «Rose Bruyère» n'a donné aucun score positif. La reconnaissance de cette

39. Ruth Bottigheimer, op. cit., p. 145. Voir plus haut, note 32, pour la référence complète.

40. Le mot «Himmel» apparaît déjà en 1810, mais il est utilisé sans connotation religieuse, comme synonyme de «ciel». 


\begin{tabular}{|c|c|c|c|c|c|c|c|c|c|}
\hline \multirow[t]{2}{*}{1510} & \multirow[t]{2}{*}{ เ\$12 } & \multicolumn{2}{|c|}{ 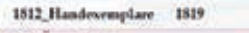 } & \multicolumn{2}{|c|}{ 1810. Hisndexranplare 1837} & \multirow[t]{2}{*}{1500} & \multirow[t]{2}{*}{ Ists } & \multirow[t]{2}{*}{1550} & \multirow[t]{2}{*}{1558} \\
\hline & & 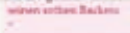 & & & & & & & \\
\hline & - & . & - & - & $=$ & - & - & - & . \\
\hline & it & ai & E & $\mathrm{Ei}$ & E & $\mathbf{E}$ & E & 百 & E \\
\hline & & & & & . & . & , & . & - \\
\hline & Au $=$ in Catr $n$ & du metrin Gianten & du metin Cater $\mathrm{A}$ & du nutin Cont $\mathrm{A}$ & 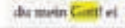 & 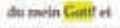 & Au nerir Conte + & An merin Ceeted & du merta Cots of \\
\hline & & & & & & & e & . & . \\
\hline & du mein Cart & du men Giart & du manc Cart & du muen Cont & damein Eiet & da mein fint & Au mein Cett & Anmen Gom & dutmina Cart \\
\hline & & & & & - & - & - & - & - \\
\hline & riofien sien & niefen vis: & Deferes ath. & pirfen aive. & neten $=$ & riden tor. & nefent vie, & antern thes & rielem aie. \\
\hline & $\mathrm{wm}$ & wow & wan & wan & nan & $\min$ & $w=$ & was & wam \\
\hline & bedan & it des & bit deat & ist das & iat des & be das & int des & in dat. & int dan \\
\hline & & & Kind & Kind & Kund & Kind & Kind & Kind & Kind \\
\hline & & & & & & & $\infty$ & ж & w \\
\hline & aldish & stiven & schent & schèn & actàn & ative & antita & stiven & scheis \\
\hline & $r$ & $r$ & $r$ & $r$ & $r$ & $r$ & $r$ & $r$ & $r$ \\
\hline
\end{tabular}

Fig. 2 : Copie d'écran d'une comparaison de dix versions de «Blanche Neige», avec le mot «Gott» en surbrillance.

\begin{tabular}{|c|c|c|c|c|c|c|c|c|c|}
\hline \multirow[t]{2}{*}{ tsto } & \multirow{2}{*}{ \$s12 } & \multicolumn{2}{|c|}{ 1s12. Hatudevemplare } & \multicolumn{2}{|c|}{ 15Ve, Handromplane IST } & \multirow{2}{*}{1860} & \multirow{2}{*}{ 1su } & 150 & \multirow[t]{2}{*}{1657} \\
\hline & & $\cdot$ & - & - & - & & & $\sigma^{*}$ & \\
\hline & Des & De & $\begin{array}{l}\text { Air astor } \\
\text { Krasentraw }\end{array}$ & Xstalle & 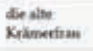 & $\begin{array}{l}\text { Sireatio } \\
\text { Kratamertiras }\end{array}$ & 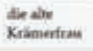 & 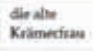 & $\begin{array}{l}\text { Ale alto } \\
\text { Krimertram }\end{array}$ \\
\hline & int & int & war & war & mar & war & wat & wat & wat \\
\hline & nimpend & nemand & nimmand & neminat & nemend & nemand & nermand & enimmunt & Nemend \\
\hline & smereen. & goweren. & & & & & & & \\
\hline & Ale Ain & als dite & ah die & alb die & als die & Als dir & alta die: & alt tite & alladin \\
\hline & & & & & & scellove & neteose & gontore & soleowe \\
\hline & Kanigin & Konigin & Kondigie & Kondigin & Kinigin & Kandigin & Kóntein & Kenigin & Karvigin \\
\hline
\end{tabular}

Fig. 3 : Copie d'écran d'une comparaison de dix versions de «Blanche Neige», montrant l'ajout de «Gottlose» en 1840.

\begin{tabular}{|c|c|c|c|c|c|c|c|c|c|}
\hline tsto & 1sม & 1512 Hendesentaler & ame tsts & 1810 Handeremp & plane 185 & 2540 & tass & 1350 & 1557 \\
\hline . mit & . Het & the & (⿻) & .60 & the & .tio & . Wet & , w & the \\
\hline ant & ont & 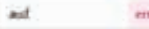 & enthich & enllich & mosh & multich & Dention & mint & mallith \\
\hline den & des & das & 40 & $4=$ & das & den & Ane & das & tas \\
\hline \multirow[t]{2}{*}{ betsise } & untume & dielvinte & wetente & sutverse & dieberes & setwents & siatvente & atabeedie & aublent: \\
\hline & & & indt wat & mele were & redte we & nectit war & necle wa & incht was & indt was \\
\hline . & , & + & & & , & . & . & is & if \\
\hline$\Delta$ & in inat & inder & und daeits & ned lavit & unel dative & unal danis & end durin & mad darin & und darin \\
\hline Men & $\log 60$ & $\log 6$ & then & but & blich & Nath & now & blieb & ntab \\
\hline$\%$ & n & $\Rightarrow$ & e & $n$ & $=$ & $\infty$ & - & - & - \\
\hline \multirow[t]{2}{*}{ liegen } & sich & sich & logenn & thegen & Liegen & likgen & Hen & tongen & lingen \\
\hline & & & betaki ach Cel & , Wvatud ocka Celt & Melat wich Cort & Detatil wich Cent & , What wat Cont & thate athe Cont & Avatal wat Coll \\
\hline
\end{tabular}

Fig. 4 : Copie d'écran d'une comparaison de dix versions de «Blanche Neige», montrant l'ajout de «befahl sich Gott» en 1819. 
absence peut aider à approfondir les recherches philologiques. Le manque de résultats conduit à se demander pourquoi la dimension chrétienne a été renforcée dans «Blanche Neige», alors que «Rose Bruyère» n'a jamais été impliqué dans les processus de christianisation. Ces histoires sont pourtant très similaires par d'autres aspects, mettant en scène une femme qui aspire à la maternité, une vilaine mégère souhaitant la mort d'une jeune fille, et une longue période de sommeil enchanté, duquel la belle se réveille pour se marier. Qu'est-ce qui a motivé les Grimm à ajouter des dimensions chrétiennes à l'un, mais pas à l'autre ? Le fait que «Rose Bruyère » est enraciné dans une tradition franco-italienne (avec des versions antérieures de Basile et de Perrault), dans laquelle les éléments chrétiens sont moins prononcés, peut fournir un élément de réponse.

Une même recherche de mots chrétiens dans «Hansel et Gretel» complique encore le tableau et soulève de nouvelles questions. On voit ici quelques opérations analogues aux observations faites dans «Blanche Neige» : en 1812, est ajoutée la mention que Dieu aidera les enfants; à partir de 1837, la sorcière est «impie »; et dès 1843 , Hans témoigne de sa foi en affirmant que Dieu n'abandonnera pas les enfants lorsqu'ils seront perdus dans la forêt. Pourtant, une seule expression est présente dans les trois éditions de 1812 à 1837 : «Gott gab es aber Gretel ein» (avec de petites variations, tel qu'indiqué ci-dessous, fig. 5).

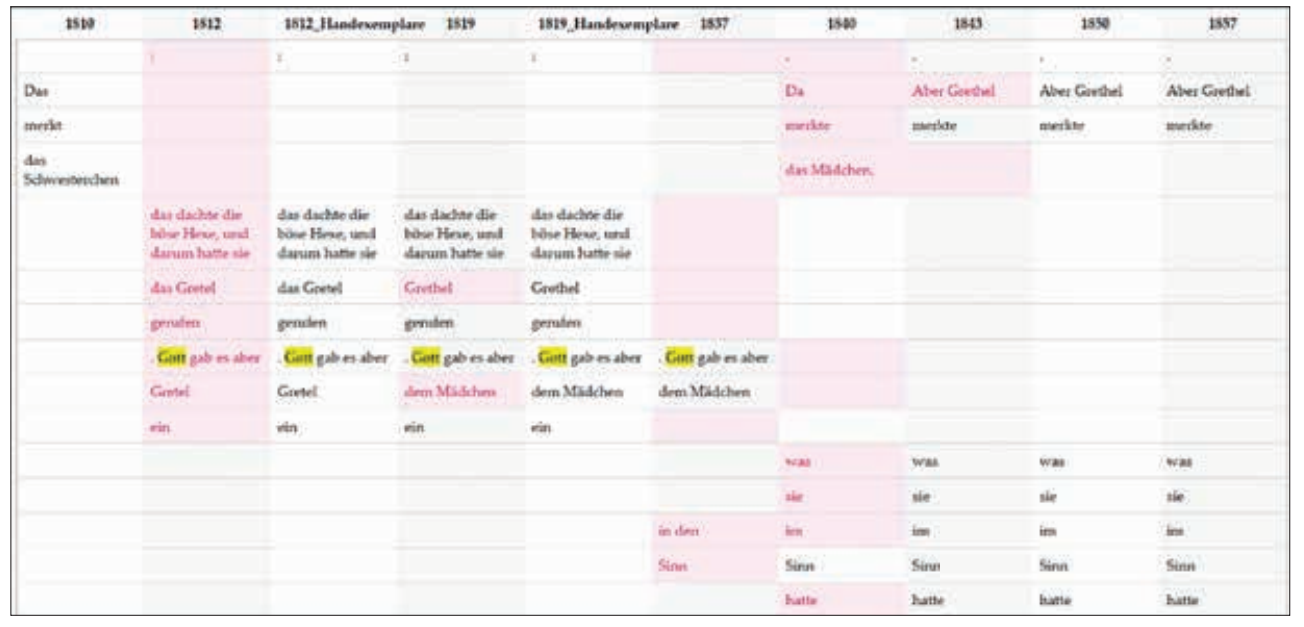

Fig. 5 : Copie d'écran d'une comparaison de dix versions de «Hansel et Gretel», avec le mot «Gott» en surbrillance.

Tandis que dans le manuscrit de 1810, Gretel elle-même comprend que la sorcière essaie de la tromper dans le but de la piéger dans le four, de 1812 à 1837, Dieu aide Gretel à comprendre ce qui se passe et ce qu'elle doit faire : faire l'ignorante à propos de la lumière du four, de sorte qu'elle puisse y pousser la sorcière elle-même. Depuis 1840, l'intervention divine est à nouveau supprimée. C'est une suppression particulièrement intéressante compte tenu de la tendance des Grimm à promouvoir le christianisme auprès de leurs jeunes lecteurs. En général, les Grimm préféraient rester vagues sur l'identité de ceux qui exécutent les châtiments. Considérons, par exemple, la scène où la vilaine reine de «Blanche Neige» est punie : 
Aber es waren schon eiserne Pantoffeln über Kohlenfeuer gestellt und wurden mit Zangen hereingetragen und vor sie hingestellt. Da mußte sie in die rotglühende Schuhe treten und so lange tanzen, bis sie tot zur Erde fiel ${ }^{41}$.

Notre traduction (littérale) : Mais les pantoufles de fer avaient déjà été placées sur le feu de charbon et transportées avec des pinces et placées devant elle. Là, elle dut mettre ses pieds dans les chaussures chauffées à vif et danser jusqu'à tomber morte sur le sol.

On pourrait se demander qui effectue ces actions. Bien que la formulation exacte change au cours des éditions, la voix passive se maintient pour servir, manifestement, un objectif clair : en ne mentionnant pas le prince, Blanche Neige, le roi, les nains ni leurs serviteurs, l'histoire peut défendre une opposition entre le bien et le mal. Même si elle n'est pas du tout aussi indulgente que la Cendrillon de Perrault, qui pardonne explicitement à ses bellessœurs, Blanche Neige et les autres personnages principaux des Grimm en général ne sont pas directement impliqués dans le châtiment des méchants, laissant ainsi leur pure bonté intacte.

Dans «Hansel et Gretel», cette même voix passive ne fonctionnerait pas, puisque l'intrigue requiert que Gretel tue la sorcière afin de libérer Hans. Faire mourir la sorcière d'une manière différente aurait privé le conte à la fois de sa scène la plus spectaculaire et du développement du personnage de Gretel.

Selon la lecture de Ronald G. Murphy, Wilhelm Grimm a réécrit le récit en «une parabole classique du voyage de l'âme humaine, de l'enfance jusqu'à la conscience du bien et du mal, le voyage du salut humain 42 ». En termes féministes, de victime passive et pleurante, Gretel devient une efficace rusée. Pourtant, l'activité de l'enfant a aussi une dimension problématique : dans le but de changer le périlleux cours des événements, Gretel doit assassiner la sorcière. En 1810, elle le fait seule; dans les éditions de 1812 à 1837, Dieu l'aide. L'intervention de celui-ci répond directement à l'appel au secours de l'enfant. Il empêche Gretel de tomber dans le piège mortel de la sorcière et fournit même peut-être une solution - à nouveau, les Grimm restent sagement vagues : la signification exacte de «es» (ça) dans «Gott gab es aber Gretel ein » n'est pas claire. L'intervention divine pourrait avoir été une solution contestée à la lumière de l'innocence de l'enfance que chérissent nombre des contemporains des Grimm : il est suggéré que Dieu pourrait avoir inspiré à l'enfant de devenir une meurtrière. Si nous considérons la première suppression du conte dans laquelle les enfants jouent au boucher, en 1819, il n'est plus guère surprenant que la scène telle qu'elle était présentée jusqu'à ce moment-là ait subi une révision. Même si Gretel ne se retient pas de tuer la sorcière - elle semble d'ailleurs n'avoir guère le choix -, Dieu au moins n'est plus partie prenante. L'ajout du qualificatif «impie» au mot «sorcière», en 1837, aide encore à justifier l'acte de la fille sans directement impliquer Dieu.

Hésitation épigénétique
L'effacement de la référence à Dieu dans la scène du four révèle un autre aspect des éditions des Grimm qu'on a tendance à ignorer. Notre collation rend particulièrement clair le fait que le processus éditorial de Wilhelm Grimm n'a pas toujours été orienté dans le même

41. Grimm, op. cit., 1857, p. 278.

42. Roland G. Murphy, The Owl, The Raven, and the Dove. The Religious Meaning of the Grimms'Magic Fairy Tales, Oxford, Oxford University Press, 2000. 
sens, mais qu'il se caractérise aussi par des moments de doute, d'hésitations et de retour en arrière. Ce n'est pas là une observation isolée, qui ne vaudrait que pour les frères Grimm ou pour la littérature pour l'enfance. L'œuvre dramatique de Samuel Beckett est un bon exemple d'hésitation épigénétique : l'auteur a révisé son œuvre chaque fois qu'il a eu l'occasion de la mettre en scène. Mais les contes de Grimm sont particulièrement intéressants sous ce rapport, leur processus de révision finissant parfois par revenir à la formulation originale. En 1840, Wilhelm Grimm revient trois décennies en arrière pour restaurer la version des événements telle qu'elle était présentée dans le manuscrit de Ölenberg. Notre comparaison présente aussi plusieurs régressions et changements que Wilhelm a considérés à un moment (selon ce qu'on peut déduire de ses annotations dans son exemplaire personnel), mais qu'il n'a finalement pas mis en œuvre dans les éditions. Par exemple, en 1812, il envisagea la possibilité de remplacer les Kuchen («biscuits») qui couvrent le toit de la maison de la sorcière dans «Hansel et Gretel» par des crêpes, mais il finit par ne rien changer.

La plupart des cas de doutes concernent la formulation, l'ordre des mots et quelques changements orthographiques (par exemple ward/wurde pour «étais»). Dans quelques cas, les révisions rejetées et les moments de doute peuvent être mis en relation avec des tendances plus générales concernant le lectorat (l'enfance) que les critiques avaient observées. Le rejet de l'expression «das lag da mit seinen rothen Backen» [elle était étendue là avec ses joues rouges], que Wilhelm ajouta à sa copie personnelle de l'édition de 1812 pour enrichir la description de Blanche Neige alors que les nains l'observaient couchée dans leur lit (fig. 6), peut être situé dans cette pratique génétique de purge du conte de toute trace d'érotisme ou de sexualité. En lieu et place, il ajoute le mot Kind («enfant») à la ligne suivante, alors que les nains admirent sa beauté - un ajout dont on peut penser qu'il désexualise la scène, du moins pour ceux qui considèrent qu'il est improbable qu'un enfant fasse l'objet d'un regard sexuel 43 .

Par son interface de visualisation, l'édition numérique facilite grandement le repérage des changements décrits ici et participe à l'affinement des résultats acquis par les travaux antérieurs ; elle stimule aussi des pratiques de recherche plus inductives, où les changements éditoriaux sont interprétés sans hypothèses préalables. Comparée à la méthode traditionnelle, consistant à traverser laborieusement des exemplaires papiers des éditions variantes, la collation numérique permet une participation plus active de la part des utilisateurs, y compris de ceux qui ne sont pas versés dans la recherche académique. Il vaut la peine de se demander si le dispositif peut donner envie à de jeunes lecteurs d'explorer la genèse des œuvres littéraires et les discours pédagogiques qui les concernent, mais dans lesquels on leur a peu donné la parole ou l'initiative. Comme le souligne Haase : «La plupart des travaux académiques sur la réception des contes de Grimm par les enfants, par exemple, n'examine pas les réactions des enfants eux-mêmes 44 », prolongeant ainsi la tendance qui caractérise l'histoire génétique
Faire participer les enfants à la critique génétique

43. Cette conception a été remise en question par des critiques de la fin du $\mathrm{xx}^{\mathrm{e}}$ siècle, comme James Kincaid, Anne Higonnet et Marah Gubar, qui relient l'idéal de l'innocence de l'enfance à une sexualisation progressive de la figure de l'enfant.

44. «Response and Responsibility in Reading Grimms'Fairy Tales», dans D. Haase (dir.), The Reception of Grimm's Fairy Tales: Responses, Reactions, Revisions, Detroit, Wayne State UP, 1993, p. 240. 
Fig. 6 : Page 242 des épreuves corrigées de Grimm pour l'édition de 1812 .

Collection de l'Université de Kassel (https://orka.bibliothek.uni-kassel.de/viewer/ object/1433243313511_1/140/)

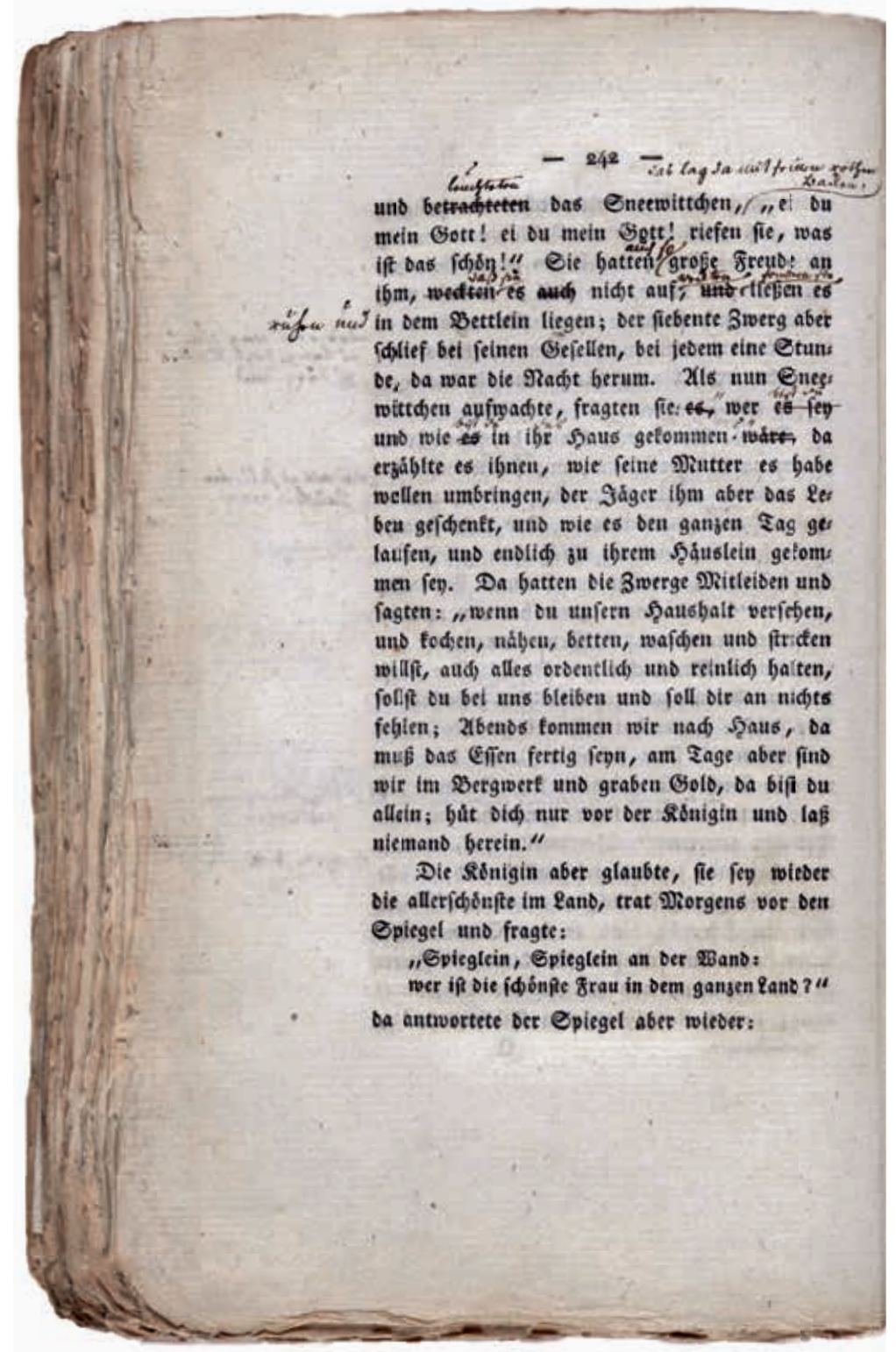

des Kinder- und Hausmärchen et qui consiste à spéculer sur les besoins des enfants et sur leurs préférences sans entendre réellement leurs voix. Une collation ergonomique rend les utilisateurs rapidement à même d'identifier les changements éditoriaux et peut ainsi servir, par exemple, dans un contexte scolaire, à stimuler des réflexions critiques et des discussions sur des sujets aussi variés que les préjugés idéologiques des Grimm, les traits génériques propres aux contes de fées, ou encore la nature et le développement des notions d'enfance et de poétique dans la littérature pour les enfants. Par ailleurs, la collation peut aider à donner aux élèves le sens de la conscience textuelle, en attirant leur attention sur le fait qu'une seule et même histoire peut exister en différentes éditions. En outre, ce genre de recherche peut encore bénéficier de l'apport des élèves et des étudiants eux-mêmes, en les intégrant dans la communauté de recherche et dans le discours critique sur la littérature pour enfants, en défenseurs de ce qu'on appelle la «recherche participative». Comme l'explique Justyna Deszcz-Tryhubczak :

L'application de la recherche participative dans les études sur la littérature jeunesse permet aux enfants de devenir des pairs contribuant à produire du savoir sur leurs lectures ayant une valeur intrinsèque semblable aux connaissances offertes les lecteurs adultes. Ce changement peut s'opérer 
en créant des espaces égalitaires dans lesquels les jeunes lecteurs peuvent être entendus par les adultes et collaborer avec eux 45 .

L'environnement numérique peut jouer le rôle d'un tel espace où les jeunes peuvent contribuer activement à l'examen critique des produits culturels dont les adultes ont été pour eux les créateurs et les médiateurs. Leur participation pourrait prendre diverses formes, comme des annotations mettant en relation des fragments de textes des Kinder- und Hausmärchen avec leurs sources ou avec des adaptations ultérieures de leurs histoires. L'implication des jeunes réclame un autre concept de l'enfant que celui fondé sur un idéal d'innocence qui informe quelques-uns des changements éditoriaux discutés plus haut. Elle présuppose plutôt un enfant compétent, bien informé, curieux et désireux de participer activement plutôt que de consommer passivement les artefacts que les adultes produisent pour lui. L'étude de leur genèse ne prive pas nécessairement les contes de Grimm de leur puissance d'enchantement, mais contribue plutôt à approfondir la compréhension que les lecteurs peuvent avoir de ces histoires et de leur succès jamais démenti de classiques pour les enfants; elle fournit une occasion unique d'étudier comment les conceptions de l'enfance façonnent, au gré de leur évolution, les artefacts culturels dédiés aux enfants - depuis l'émergence de la littérature pour enfants jusqu'à aujourd'hui.

Article traduit par Rudolf Mahrer

45. Justyna Deszcz-Tryhubczak, «Using Literary Criticism for Children’s Rights: Toward a Participatory Research Model of Children's Literature Studies», The Lion and the Unicorn, n 2, 2016, p. 217.

DiRK VAN Hulle est professeur de littérature anglaise à l'université d'Anvers et directeur du Centre pour la génétique des manuscrits. Ses publications comprennent Textual Awareness, Modern Manuscripts, Samuel Beckett's Library (with Mark Nixon), The New Cambridge Companion to Samuel Beckett, James Joyce's Work in Progress, plusieurs volumes de la série "Making of" (Bloomsbury) et des éditions génétiques au sein du Beckett Digital Manuscript Project, y compris la Beckett Digital Library.

dirk.vanhulle@ua.ac.be

VANESSA JOOSEN est professeure associée de littérature anglaise et de littérature pour enfants à l'université d'Anvers. Elle est auteure, entre autres, de Critical and Creative Perspectives on Fairy Tales et de Adulthood in Children's Literature. Elle a édité Grimm's Tales Around the Globe: The Dynamics of their International Reception (with Gillian Lathey) et Connecting Childhood and Old Age in Popular Media.

vanessa.joosen@uantwerpen.be

VinCENT NEYT est chercheur au Centre pour la génétique des manuscrits à l'université d'Anvers. Spécialisé dans les éditions savantes numériques, il est développeur technique du Beckett Digital Manuscript Project. Il a publié dans Literary and Linguistic Computing et dans Digital Scholarship in the Humanities.

vincent.neyt@gmail.com 


\section{Épigenèse et littérature pour enfants}

Le phénomène de l'épigenèse (la continuation de la genèse après la publication) est particulièrement pertinent en littérature pour enfants. Pour développer cette thèse nous étudions le cas des contes des frères Grimm à la lumière de la conception de l'enfance du début du XIXe siècle. L'objectif est de montrer (1) que des conceptions opposées de l'enfance peuvent avoir un effet sur des révisions épigénétiques, et combien les négociations au cours de ce processus peuvent être complexes; (2) comment des outils numériques peuvent aider les chercheurs en génétique à développer leurs observations concernant les étapes de l'épigenèse des contes de Grimm, qui ne comprend pas moins de sept (grandes) éditions; et (3) comment ces outils peuvent aider à introduire la genèse de Die Kinder- und Hausmärchen auprès de ceux-là mêmes qui sont les sujets de ce discours : les jeunes lecteurs.

In this article, we argue that the phenomenon of epigenesis (the continuation of the genesis after publication) is particularly relevant to children's literature. To corroborate this argument, we take the Grimm brothers'fairy tales as a case study and examine them in the light of early-nineteenth-century views on childhood. The aim is to demonstrate (1) how conflicting concepts of childhood can influence epigenetic revisions, and how complex the negotiations in this process may be; (2) how digital tools (notably automatic collation) can assist genetic critics in developing their insights into the epigenetic stages of the Grimm tales, which involve no less than seven (large) editions; (3) how digital tools may help to provide access to the genesis of Die Kinder- und Hausmärchen to the very subjects of this discourse: the young readers themselves.

Das Phänomen der Epigenese (Weiterführung der Genese nach der Veröffentlichung) ist für die Kinder- und Jugendliteratur besonders relevant. Um diese These zu entwickeln, werden hier die Märchen der Brüder Grimm im Lichte der Kindheit am Anfang des 19. Jahrhunderts untersucht. Das Ziel ist es zu zeigen, dass (1) entgegengesetzte Konzeptionen von Kindheit sich auf epigenetische Revisionen auswirken können und wie komplex die Auffassungen im Zuge dieses Prozesses mitunter sind; (2) wie digitale Werkzeuge den Forschern im Bereich der Textgenese helfen können, ihre Beobachtungen über die Stufen der Epigenese von Grimm's Märchen so anzustellen, dass sie nicht weniger als sieben (große) Editionen umfasst; und (3) wie diese Werkzeuge hilfreich sein können, um in die Genese von den Kinder- und Hausmärchen einzuführen, und zwar gerade für jene, die darin vorkommen: die jungen Leser selbst.
El fenómeno de la epigénesis (prosecución de la génesis después de la publicación de la obra) es particularmente pertinente en literatura para niños. Para desarrollar esta tesis estudiaremos el caso de los cuentos de los hermanos Grimm a la luz de la concepción de la infancia de comienzos del siglo XIX, con el objetivo de mostrar (1) que concepciones opuestas de la infancia pueden afectar las revisiones epigenéticas y cuan complejas pueden ser las negociaciones a lo largo de ese proceso; (2) como las herramientas digitales pueden ayudar a los investigadores en génesis a desarrollar sus observaciones relativas a las etapas de la epigénesis de los cuentos de Grimm, que comprende nada menos que siete (grades) ediciones; y (3) como estas herramientas pueden contribuir a introducir la génesis de Die Kinder- und Hausmärchen entre aquellos que son los sujetos mismos de este discurso: los jóvenes lectores.

F enómenos de epigénese (prolongamento da génese após a publicação) são particularmente pertinentes na literatura para crianças. Em apoio desta ideia, estudamos o caso dos contos dos irmãos Grimm, à luz do conceito de infância no início do século XIX. O objetivo é mostrar que (1) concepções opostas sobre a infância podem ter efeito sobre revisões epigenéticas, implicando complexas negociações; (2) e que instrumentos digitais podem ajudar os geneticistas a desenvolver as suas observações sobre as etapas epigenéticas dos contos de Grimm, que compreendem não menos de sete edições importantes; e (3) como esses instrumentos podem ajudar a apresentar a génese de Die Kinder- und Hausmärchen junto daqueles que são sujeito deste discurso: os jovens leitores.

I 1 fenomeno dell'epigenesi (la continuazione della genesi dopo la pubblicazione) concerne in particolar modo la letteratura per l'infanzia. Per sviluppare questa tesi, l'articolo studia il caso dei racconti dei fratelli Grimm alla luce della concezione dell'infanzia all'inizio del XIX secolo. L'obiettivo è dimostrare 1) in che modo concezioni opposte dell'infanzia possono influenzare le revisioni epigenetiche ( $\mathrm{e}$ in che misura le negoziazioni durante questo processo possono essere complesse); 2) in che modo gli strumenti digitali possono aiutare i ricercatori a sviluppare le loro osservazioni intorno alle tappe dell'epigenesi dei racconti di Grimm, che contano almeno sette (grandi) edizioni; e 3) come questi strumenti possono aiutare a introdurre la genesi di Kinder- und Hausmärchen presso i soggetti stessi di questo discorso: i giovani lettori. 